\title{
ESTADO DEL ARTE DE LA COMUNICACIÓN MÓVIL EN ESPAÑA. APORTACIONES REALIZADAS Y RETOS DE FUTURO
}

\author{
Carmen Costa- Sánchez ${ }^{1}$ : Universidade da Coruña. España \\ carmen.costa@udc.es
}

\section{RESUMEN}

La comunicación móvil se está convirtiendo en una nueva necesidad comunicativa de la Sociedad de la Información. El año 2010 se ha convertido en un año de referencia en nuestro país, tanto por el incremento en las ventas de dispositivos móviles, como por la creación de sistemas operativos y el éxito de las aplicaciones para dichas plataformas. En el presente artículo, nos preguntamos cómo se está abordando este fenómeno desde las Ciencias de la Comunicación: desde qué perspectivas, con qué objetivos y con qué resultados. Lo que se pretende es establecer un estado del arte de la investigación sobre comunicación y dispositivos móviles en España para poder definir nuevas líneas de acercamiento a este fenómeno que se ha convertido en un nuevo ámbito de estudio de creciente interés.

PALABRAS CLAVE: Comunicación móvil - Movilidad- Dispositivos móviles España - Investigación

\section{STATE OF THE ART OF THE MOBILE COMMUNICATION IN SPAIN. CONTRIBUTIONS REALISED AND CHALLENGES OF FUTURE}

\begin{abstract}
The mobile communication has become a new communicative need for the Information Society. The year 2010 has been a year of reference in our country, so much by the increase in the sales of this type of devices, as by the creation of operating systems and of applications for these new platforms. In the present work, we want to know how this phenomenon is being tackled from Communication

\footnotetext{
${ }^{1}$ Autor correspondiente

Carmen Costa- Sánchez: Doctora. Profesora Ayudante. Universidade da Coruña. A Coruña, España.

Correo: carmen.costa@udc.es
} 
Sciences: from which perspectives, with what objective and with which results. We pretend to establish a state of the art of the investigation on communication and mobile devices in Spain to be able to define new lines of research on this phenomenon that has turned into a new field of study of increasing interest.

KEY WORDS: Mobile Communication - Mobility - Mobile Devices - Spain - Research

\section{INTRODUCCIÓN}

\subsection{El éxito comercial de la telefonía y de los dispositivos móviles en España. Datos contextuales}

El destacado crecimiento en las ventas de teléfonos y de dispositivos móviles en nuestro país parecen apuntar a una necesidad social creciente por estar permanentemente comunicados con nuestro entorno (con nuestra red social), especialmente en el caso de las nuevas generaciones (o los llamados nativos digitales).

La tasa de penetración de la telefonía móvil en España ha alcanzado en 2010 un $109,7 \%$, cuatro décimas más que en 2009 , pero un $37,6 \%$ más que en el inicio de la década.

\section{Penetración}

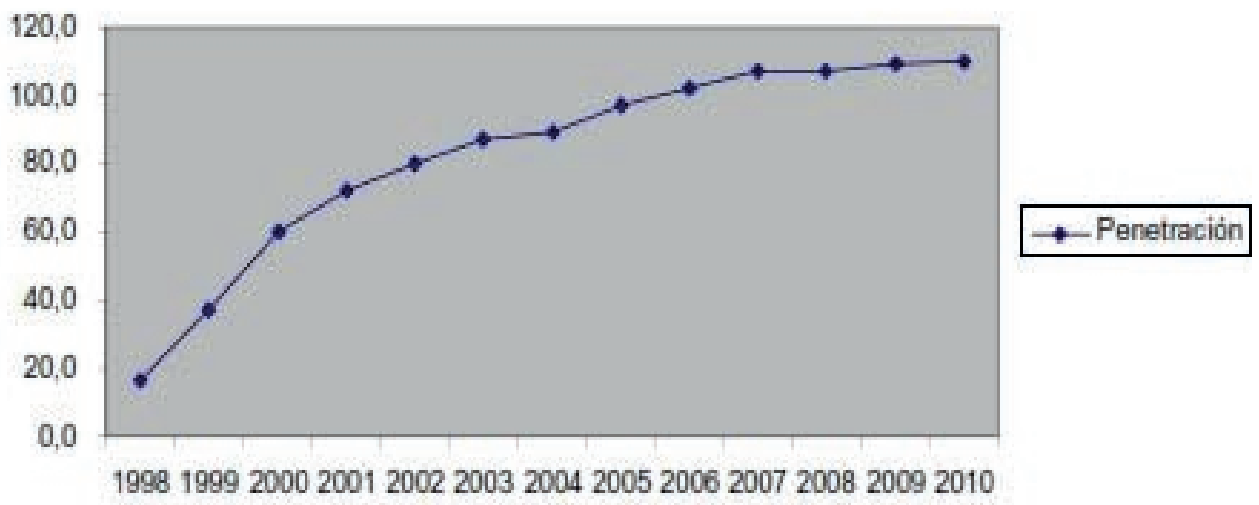

Figura 1. Tasa de penetración de la telefonía móvil sobre la población (Líneas/100 habitantes). Fuente: Informe Anual 2010. Comisión del Mercado de las Telecomunicaciones

Cabe destacar que el año 2010 ha sido un año de referencia, en lo que a dispositivos móviles se refiere, debido a tres aspectos fundamentales que se recogían en el Informe La sociedad de la información en España 2010 de la Fundación Telefónica:

a)

En primer lugar, la consolidación de los smartphones con capacidad de acceso a Internet, con un crecimiento del $50 \%$ en cuanto a unidades vendidas durante el último año. En el caso concreto de España, las ventas de smartphones se incrementaron en un $90 \%$ durante el último año. Un consumo muy superior 
entre la juventud, en concreto, la penetración de los smartphones entre la juventud europea promedia un $28 \%$ mientras que la penetración en el target adulto en Europa es del 27\%.

b)

En segundo lugar, la evolución espectacular del sistema Android apadrinado por Google frente al desarrollo de otros sistemas operativos.

c)

En tercer lugar, la adopción generalizada de interfaces táctiles con la intención de hacer más intuitivo su uso y navegación, destacando la venta de tablets (como por ejemplo, la llegada al mercado español del iPad).

Según la Encuesta sobre Equipamiento y Uso de Tecnologías de Información y Comunicación en los Hogares correspondiente al año 2010 (y elaborada por el Instituto Nacional de Estadística ${ }^{2}$ ), la presencia de ordenadores portátiles en los hogares españoles prácticamente iguala al número de PC's (un 42,5\% frente a un $50,1 \%)$; la penetración del teléfono móvil aumenta 1,1 puntos hasta llegar a estar disponible en el 94,6\% de los hogares.

Y no sólo los soportes preparados para la movilidad están cada vez más extendidos, sino que estos permiten llevar la conectividad a cualquier parte, generando una accesibilidad no limitada a nivel espacial y/o temporal. De hecho, en datos del INE, los principales equipos disponibles en las viviendas para conectarse a Internet son el ordenador de sobremesa (el $72,0 \%$ de las viviendas), el portátil (61,1\%) y el teléfono móvil $(25,4 \%)$ y se subraya que aumentan los accesos mediante otro tipo de dispositivo (agendas electrónicas, PDAs, televisión o videoconsola).

Tal y como explica García Hervás (2010), con una base mundial de más de 4.600 millones de clientes a principios de 2010 y una tasa de penetración del 113,9 por ciento en España, superando los 54,4 millones de líneas, las comunicaciones móviles permiten potenciar la utilidad de las redes que se interconectan a ella, principalmente Internet, consiguiendo una sinergia que combina movilidad con el catálogo de servicios multimedia interactivos existente en la Red.

Los datos de ventas de tablets en nuestro país corroboran la entrada en una nueva etapa de comunicación en movilidad y las tendencias de futuro apuntan a una mayor penetración de smartphones y tabletas en nuestro país (según pronostica el Informe de la Industria de los Contenidos Digitales 2011).

El incremento cuantitativo, por tanto, demostrable en términos de mercado, implica el desarrollo de prácticas nuevas de comunicación, de interacción, de entretenimiento, de información, etc. que suponen cambios importantes a diversos niveles comunicativos, que todavía hay que analizar:

\footnotetext{
2 http://www.ine.es
} 
"La telefonía móvil ha supuesto una radical transformación en los ritos sociales de la interacción (Gesser, 2004), en la administración de los tiempos cotidianos, en los procesos de gestión de las dinámicas grupales y organizacionales y en la gestión de fronteras e interconexiones entre diferentes ámbitos personales (trabajo, ocio, familia, grupos primarios" (Aguado y Martínez, 2006, p. 321).

Las nuevas potencialidades de uso que proponen los "nuevos media" pasan por las siguientes transformaciones sociales:

Primeramente, la conectividad deja de estar vinculada al espacio de forma que la accesibilidad se vuelve continua (always on). De ahí la importancia del móvil como dispositivo personal, como un complemento de la persona contribuyendo a la proyección de identidad y de marca personal y permitiendo el consumo y producción de contenidos en cualquier lugar y a cualquier hora.

Además, el móvil se convierte en un complemento de uso en el llamado ocio intersticial, que se desarrolla fundamentalmente en los tiempos de espera y de traslados, en los intervalos entre actividades cotidianas.

Por otra parte, el acceso a la Web amplía la finalidad de comunicación interpersonal (de uno a uno), a comunicación de masas (del medio a los ciudadanos), a comunicación grupal (de redes o entre pares) y de uno a muchos (del individuo a su red de contactos). La unidireccionalidad se vuelve bidireccionalidad o incluso multidireccionalidad gracias a las herramientas de la Web participativa.

Teniendo en cuenta los aspectos cuantitativos y cualitativos ya comentados, resulta de interés realizar un análisis acerca de la producción científica realizada en nuestro país sobre este fenómeno. El objeto de la presente aportación es el de proponer una revisión de la producción científica que los investigadores españoles han realizado para conocer así cuales han sido los principales focos de interés, así como las perspectivas desde las que sería interesante que se abordase el fenómeno.

\section{METODOLOGÍA}

Según explicaban Aguado y Martínez (2006), el análisis del impacto social de la telefonía móvil lleva entre 10 y 15 años de retraso respecto de su implantación puesto que es desde el año 2000 que forma parte de la agenda de investigación fundamentalmente de los países del ámbito anglosajón y del norte de Europa.

El propósito de la presente investigación es el de averiguar su grado de implantación en la agenda de investigación española. Para ello, se ha recurrido a la revisión de los recursos bibliográficos publicados en España sobre comunicación móvil. En concreto, se han recopilado y analizado todos los artículos indexados en Dialnet que respondiesen a las palabras clave "comunicación móvil", "comunicación + movilidad" y "comunicación + dispositivos móviles". 
Se ha escogido la base de datos Dialnet por ser la única de acceso libre y gratuito que recoge tanto artículos de revistas, como ediciones de actas, tesis doctorales y libros en el ámbito de la literatura científica hispana ${ }^{3}$. Los resultados han sido filtrados posteriormente para comprobar que el objeto de estudio era el correspondiente así como para restringir su ámbito geográfico de edición al territorio español. No obstante, se han descartado los estudios técnicos o exclusivamente tecnológicos y se han revisado aquellos con mayor relación con el campo de la Comunicación.

Los resultados hallados se presentan cuantitativamente en la siguiente tablaresumen:

Tabla 1. Resumen de los documentos científicos registrados en Dialnet.

\begin{tabular}{|l|l|l|l|l|l|}
\hline Resultados & \multirow{2}{*}{$\begin{array}{c}\text { Términos de } \\
\text { búsqueda }\end{array}$} & \multicolumn{3}{|l|}{ Tipo de recurso encontrado } \\
\cline { 3 - 6 } & "comunicación móvil" & 14 & 92 & 1 & 5 \\
\hline 112 & Libro & Artículo & Tesis & Actas \\
\hline 11 & $\begin{array}{l}\text { "comunicación + } \\
\text { movilidad" }\end{array}$ & 0 & 10 & 0 & 1 \\
\hline 8 & $\begin{array}{c}\text { "comunicación + } \\
\text { dispositivos moviles }\end{array}$ & 0 & 8 & 0 & 0 \\
\hline
\end{tabular}

La revisión de los recursos encontrados así como el análisis de los mismos nos permitirá tener una visión de conjunto de las principales líneas de investigación seguidas en España, así como de las principales aportaciones realizadas.

\section{ANÁLISIS Y DISCUSIÓN}

Las investigaciones realizadas acerca de la comunicación móvil en España han fructificado sobre todo en artículos científicos dado que las revistas científicas son el soporte de difusión que se actualiza de forma periódica y procura renovar temas para la llamada a colaboraciones de cada número (los llamados 'call for papers').

El primero de los artículos sobre esta temática registrado en la base de Dialnet data del año 2001. Podemos concluir por tanto, que es un área de estudio relativamente reciente en nuestro país (hace apenas una década que se plantea como objeto de investigación), que va adquiriendo mayor interés a la sombra del éxito comercial del fenómeno.

Tampoco ha generado todavía suficientes tesis doctorales, aunque probablemente sea una tendencia que se vea invertida en los próximos años al amparo de la relevancia social y éxito de mercado adquirido.

\footnotetext{
${ }^{3}$ http:/ / www.fundaciondialnet.es/dialnet/descripcion/
} 
Cabe destacar también que la terminología "comunicación móvil" va sedimentando como la denominación de este ámbito de estudio, definido por el empleo de tecnologías móviles que permiten al usuario no sólo la comunicación interpersonal, sino el acceso a la Red y a todas sus posibilidades, con las transformaciones que ello implica.

En el año 2002, la Revista Estudios de Juventud, plataforma de publicación del Injuve, el organismo público adscrito al Ministerio de Sanidad, Política Social e Igualdad, da a conocer diversos trabajos relacionados con la comunicación móvil. En ese año la revista dedica el número a la temática Juventud y Telefonía móvil, publicando diversos artículos sobre el empleo de los teléfonos móviles por parte del público infanto-juvenil.

En general, la relación y dinámicas de uso de los dispositivos móviles por parte del target juvenil ha sido el más estudiado desde las Ciencias de la Comunicación, así como desde la Psicología o Educación. Desde las Ciencias de la Comunicación se ha optado por una línea que entronca con la Teoría de Usos y Gratificaciones, que centra su interés en intentar comprender las razones concretas que conllevan el empleo de los medios de comunicación con variables como las necesidades, los propósitos, los beneficios y las consecuencias del uso de los medios de comunicación (Faber, 2000; Rubin, 1994). El empleo del móvil por parte de niños, adolescentes y jóvenes en general ha sido el objeto de estudio más prolífico en nuestro país a nivel de resultados de investigación publicados. Tal y como explica Castells et al. (2007, p.208), "los jóvenes adolescentes, los de veinte y treinta años, no sólo conforman la mayor proporción de usuarios, sino que también son los primeros en adoptarla, los que inventan usos no previstos por los diseñadores de dicha tecnología".

Desde una óptica de conjunto, una revisión de la bibliografía publicada sobre este fenómeno ha aportado luz sobre las siguientes cuestiones:

La existencia de diferencias en el uso en función del género y del tramo de edad. En cuanto a la edad, destacaría el uso de SMS en tramos de corta edad y especialmente destacable en el caso de familias de hijos únicos, al tiempo que una preferencia por las llamadas de voz más que por la mensajería escrita en tramos de edad más mayores. En cuanto al género, las chicas preferirían hablar a escribir para convertirse en soportes de la red social; en cualquier caso, ambos lo emplearían para reforzar su identidad individual y colectiva y servir de frontera frente a los padres (Revista Estudios de Juventud $n^{\circ}$ 57, 2002). No obstante, los estudios indicados se produjeron en un contexto tecnológico y social en el que las redes sociales todavía no habían irrumpido en las dinámicas relacionales de los jóvenes, como ha comenzado a producirse especialmente desde el bienio 2008-20094 .

\footnotetext{
${ }^{4}$ Las redes sociales e Internet móvil: hitos tecnológicos del 2009”, disponible en El País, 22/12/2009, http://www.elpais.com/articulo/economia/redes/sociales/Internet/movil/hitos/tecnologicos/2009/ elpepueco/20091222e
} 
a)

Menos numerosos son los estudios centrados en la mujer como otro de los targets estudiados, aunque ya existen algunas experiencias al respecto. En este sentido, basándose en una metodología cualitativa de los focus group, se ha concluido que "las mujeres prefieren asegurar las funcionalidades básicas, la practicidad y el diseño, centrando sus usos en acciones comunicativas personales y cercanas" (Martínez, Aguado y Tortajada, 2009, p. 30).

b)

temáticas generadoras de interés desde las Ciencias de la Comunicación ha sido la vinculada a la comunicación corporativa y también al marketing; por lo tanto, desde una óptica de potencialidades de uso de la nueva tecnología al servicio de la estrategia empresarial o comercial, acuñando una nueva terminología derivada del uso de dispositivos móviles como soporte para acciones con dicha finalidad, la del "marketing móvil" (Feijoo González et al., 2010; Cano, 2006; De Bernardo González et al., 2007).

c)

Desde el ámbito periodístico, la movilidad de la información y el uso de los dispositivos para informarse también ha sido objeto de interés. En ese sentido, se ha investigado la situación de presente y de futuro de la televisión en el móvil. Se ha concluido que la televisión móvil asentará su presencia en el mercado sólo si se sortean algunos de los obstáculos existentes, se pone el foco en el posible usuario (y no en el producto) y se cuenta con cierto apoyo institucional (Feijoo et al., 2010).

d) situación relativa a la prensa o más concretamente a los cibermedios españoles (Costa Sánchez y Piñeiro Otero, 2010). Se ha podido concluír que todavía queda un camino largo por delante para explotar el sector informativo en los soportes móviles, aunque a lo largo de este 2011 hayan fructificado iniciativas de las principales empresas periodísticas españolas.

e)

También desde la óptica del e-learning se han realizado aproximaciones a lo que se ha dado en llamar el mlearning o aprendizaje móvil, etc., fenómeno reciente que propondría el empleo de los dispositivos móviles con fines educativos. En esta línea se han analizado los potenciales usuarios, los retos y las barreras que se están encontrando en el desarrollo de dicha finalidad, fundamentalmente, ergonómicas, de escasa oferta de contenidos y de alto coste económico de los propios dispositivos (Sotelo González, 2009).

f)

La capacidad de movilización social ha sido, desde una perspectiva más bien política otro eje de interés. Las llamadas smart mobs están constituidas por grupos de personas que utilizan teléfonos móviles para organizar acciones colectivas. La participación política o la movilización ciudadana gracias al empleo de estos soportes supone por tanto otra línea de interés.

En general, las líneas de investigación sobre comunicación móvil en nuestro país son incipientes y deben afrontar la dificultad de desarrollarse en un entorno cambiante, 
tanto desde el punto de vista tecnológico, por cuanto a los nuevos dispositivos y/o a sus nuevas potencialidades, como sociológico, en lo referente al desarrollo de nuevos hábitos de uso y prácticas de interacción.

Al permitir la conectividad a la Red, always on, los dispositivos móviles potencian el interés suscitado por las nuevas prácticas de interacción a nivel interpersonal y grupal gracias a Internet.

Por otra parte, como medio de comunicación aglutinador para los ámbitos privado, profesional/laboral y social, y como medio para informar (se), la riqueza y variedad de mensajes y de finalidades de uso son múltiples, lo que abre también continuos campos de interés.

\section{CONCLUSIONES}

La comunicación móvil presenta para el investigador el reto de enfrentarse a un ámbito por explorar, pero que está en continua transformación. Grosso modo, las líneas de investigación iniciadas en el marco de las Ciencias de la Comunicación son:

a)

Estudios desde la perspectiva de Usos y Gratificaciones, centrados sobre todo en el target infantil, adolescente y juvenil.

b)

Estudios desde la perspectiva de las Industrias Culturales y, en concreto, acerca de la producción de contenidos para telefonía y otros dispositivos móviles.

c)

Estudios desde la Comunicación Corporativa y de Marketing, por tanto entendiendo el móvil como un nuevo canal de comunicación al servicio de los objetivos institucionales y de negocio de la empresa.

d)

Estudios desde la perspectiva del Aprendizaje o E-learning, en este caso, M-learning, que buscan aplicar las nuevas funcionalidades de los dispositivos a las mejoras didácticas de los procesos de aprendizaje.

e)

Estudios desde la perspectiva del Periodismo, centrados especialmente en el grado de adaptación de los medios de comunicación a la Web móvil.

f)

Estudios desde la perspectiva de la ciberdemocracia o ciudadanía 2.0, por tanto,como un nuevo canal de comunicación al servicio de las acciones de movilización de la ciudadanía, con efecto exponencial.

La reflexión sobre lo que hay o sobre lo que empieza a haber ha de permitirnos ahora detenernos sobre aquellas líneas de investigación que podrían resultar de interés de cara a revertir en una mejora social.

Siguiendo el modelo de Lasswell -quien dice qué a quién por qué canal y con qué efectos- podemos apuntar los campos de interés desde los que acercarse a este fenómeno desde la óptica de las Ciencias de la Comunicación. 
Desde la perspectiva del emisor (QUIEN), interesa el posicionamiento de las operadoras, de los productores de contenidos y de las organizaciones -empresas e instituciones en su sentido más amplio- que empleen dicha tecnología y de cómo lo empleen para relacionarse con sus públicos. Pero también, derivado de la multidireccionalidad del fenómeno, de las intenciones, dinámicas, hábitos y finalidades con que la ciudadanía los utilice. La figura del prosumidor de contenidos que no sólo consume sino que también los produce- está también vinculada a la denominada mobile web 2.0.

Desde el análisis de los contenidos (QUÉ), resulta de especial relevancia el conocer las aplicaciones, el tipo de contenidos multimedia ofertados y aquellos para los que las características de los dispositivos pudiesen resultar adecuados. También los formatos de dichos contenidos deben adecuarse al nuevo medio. Formatos y lenguajes que se adapten a las características de los nuevos medios.

El estudio de las audiencias o usuarios (A QUIÉN) es por el momento la línea de investigación más abordada, pero hay que tener en cuenta la gran diversidad de públicos que emplean la tecnología móvil con distintas finalidades. El público infantil y juvenil (sobre todo el adolescente), ha sido el más estudiado, pero quedan por confirmar tendencias de uso desde la perspectiva sociológica. En cuanto al género, también se detecta la carencia de estudios en este sentido, que permitan establecer si existen diferencias sustanciales entre usuarios y usuarias.

El canal, que en este caso llamaremos MEDIO, influye notablemente en el tipo de empleo a realizar. En la actualidad, el término teléfono móvil designa apenas una parcela de una realidad mucho más amplia: PDA's, iPads, tablets, e-books, etc. El tipo de dispositivo, con sus características -limitaciones y potencialidades- condiciona el uso y los hábitos.

En cuanto a las teorías de los EFECTOS, como ya se ha comentado, la teoría de usos y gratificaciones ha proporcionado una óptica útil para acercarse al fenómeno. No obstante, quizás sea demasiado pronto para aventurar efectos de un fenómeno que se encuentra en creciente expansión.

\section{REFERENCIAS}

Aguado, J.M. \& Martínez, I. (2006). El proceso de mediatización de la telefonía móvil: de la interacción al consumo cultural. ZER, (20):319-343.

Cano, J. LL. (2006). Las tecnologías emergentes como nuevo soporte de marketing: Mobile Marketing. MK: Marketing + Ventas, (211): 32-35.

Castells, M., Fernández-Ardévol, M., Linchuan Qiu, J. \& SY, A. (2007). Comunicación móvil y sociedad. Una perspectiva global, Recuperado el 20 de Noviembre de 2011, de www.eumed.net/libros/2007c/312/ 
Costa Sánchez, C. \& Piñeiro Otero, T. (2010). Prensa en la cuarta pantalla: movilidad de la información del papel al bolsillo. El profesional de la información, 19, (6): 632-636.

De Bernardo González, C. M. \& Priede Bergamini, T.(2007). Marketing móvil: una nueva herramienta de comunicación: análisis y nuevas perspectivas para el mercado español. A Coruña: Netbiblo.

Faber, R. J. (2000). The urge to buy: A uses and gratifications perspective on compulsive buying. En S. Ratneshwar, D. G. Mick \& C. Huffman (Eds). The why of consumption (pp. 177-196). London: Routledge.

Feijoo González, C.; Gómez Barrosos, J. L.;\& Martínez Martínez, I. (2010). Nuevas vías para la comunicación empresarial: publicidad en el móvil. El profesional de la información, 19, (2): 140-148.

Barroso, J. L. \& Ramos Villaverde, S. (2010). Medios de comunicación en internet móvil: la televisión como modelo aún pendiente de éxito. El profesional de la información, 19, (6): 637-644.

Fundación Telefónica (2010). La sociedad de la información en España 2010, Ariel, de http://e-libros.fundacion.telefonica.com/sie10/aplicacion_sie/ParteA/datos.html

Martínez Martínez, I.; Aguado Terrón, J. M. \& Tortajada, I. (2009). Movilizad@s: Mujer y comunicaciones móviles en España. Feminismo /s: revista del Centro de Estudios sobre la Mujer de la Universidad de Alicante, (14): 15-34.

Rubin, A. M. (1994). Uses, gratification and media effects research. En J. Bryant \& D. Zillman (Eds). Media effects: Advances in theory and research (pps. 281-301). Hillsdale. NJ: Erlbaum.

Scolari, C. A. et al. (2009). Comunicación móvil: actores y producción de contenidos en Cataluña. Comunicación y Sociedad, 22, (2): 159-185.

Sotelo González, J. (2009). Una academia en cada iPhone. Telos. Cuadernos de comunicación e innovación, (81): 122-128.

\section{Carmen Costa- Sánchez}

Doctora en Comunicación por la Universidade de Santiago de Compostela. Su ámbito de investigación incluye las nuevas plataformas de comunicación multimedia y el móvil, línea en la que ha desarrollado diversas publicaciones. En la actualidad es profesora en la titulación de Comunicación Audiovisual de la Universidade da Coruña. 